Supplementary Information for

\title{
Single-Coil, Multisample, Proton Relaxation Method for Magnetic Relaxation Switch Assays
}

Thomas J. Lowery*, Robert Palazzolo, Susanna M. Wong, Pablo J. Prado, Sonia Taktak

286 Cardinal Medeiros Ave. Cambridge, MA 02141

\section{Contents}

Figures S-1, S-2, S-3, S-4 


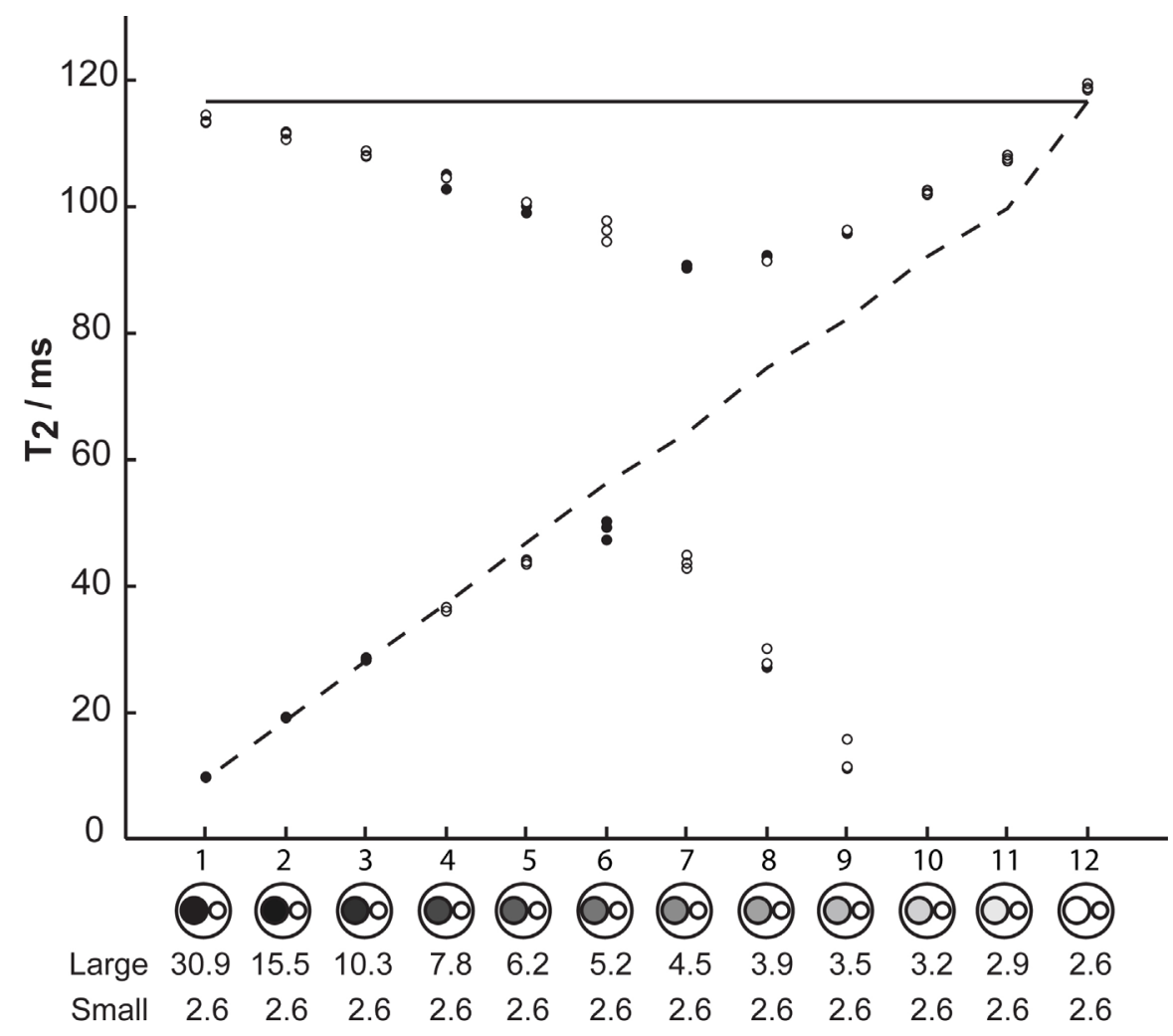

Figure S-1. Plots of $\mathrm{T}_{2}$ fit coefficients obtained for the series of $\mathrm{CuSO}_{4}$ standard solutions shown in Figure 3. The small chamber of the plastic cartridge was filled with $2.6 \mathrm{mg} / \mathrm{mL} \mathrm{CuSO}$ and the large chamber was filled with $\mathrm{CuSO}_{4}$ solutions ranging in concentration from $30.9 \mathrm{mg} / \mathrm{mL}$ to $2.6 \mathrm{mg} / \mathrm{mL}$. $\mathrm{T}_{2}$ values of these solutions were obtained individually and were plotted for the large (dotted lines) and small (solid lines) chambers. Fitting relaxation curves obtained from both chambers simultaneously with Equation (2) resulted in the $T_{2}$ coefficients (open and closed circles) that roughly follow the actual $\mathrm{T}_{2}$ values for concentrations $30.9-10.3 \mathrm{mg} / \mathrm{mL} \mathrm{CuSO}_{4}$, after which the values deviate significantly from actual $\mathrm{T}_{2}$ values. All data were acquired, analyzed and plotted in triplicate. 
(a)

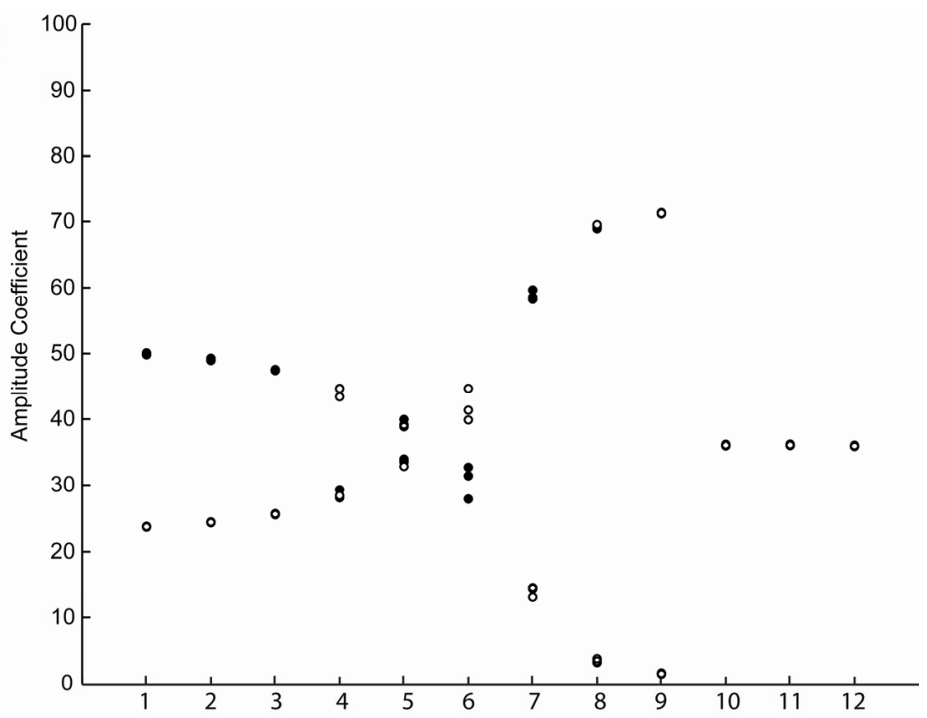

(b)

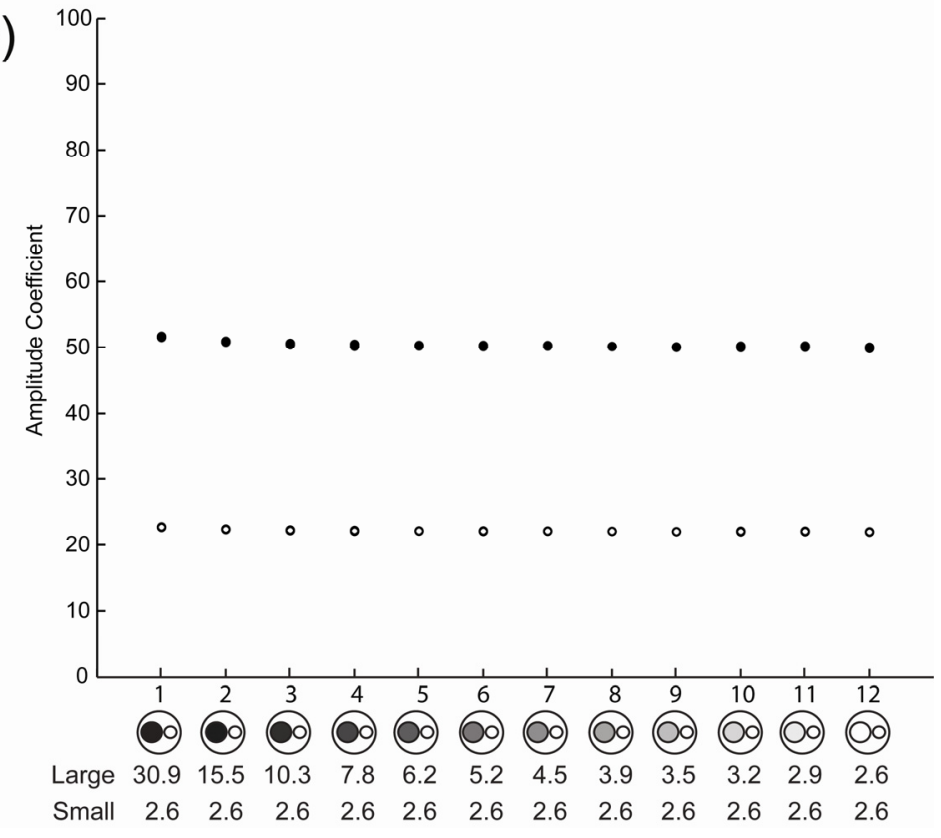

Figure S-2. Plots of Amplitude fit coefficients obtained for the same series of $\mathrm{CuSO}_{4}$ standard solutions shown in Figures 3 and S-1. Amplitude values for fits using Equation (2) are erratic whereas amplitudes for fits using Equation (3) correspond to the ratio of relative volumes for the small and large chambers. All data were acquired, analyzed and plotted in triplicate. 

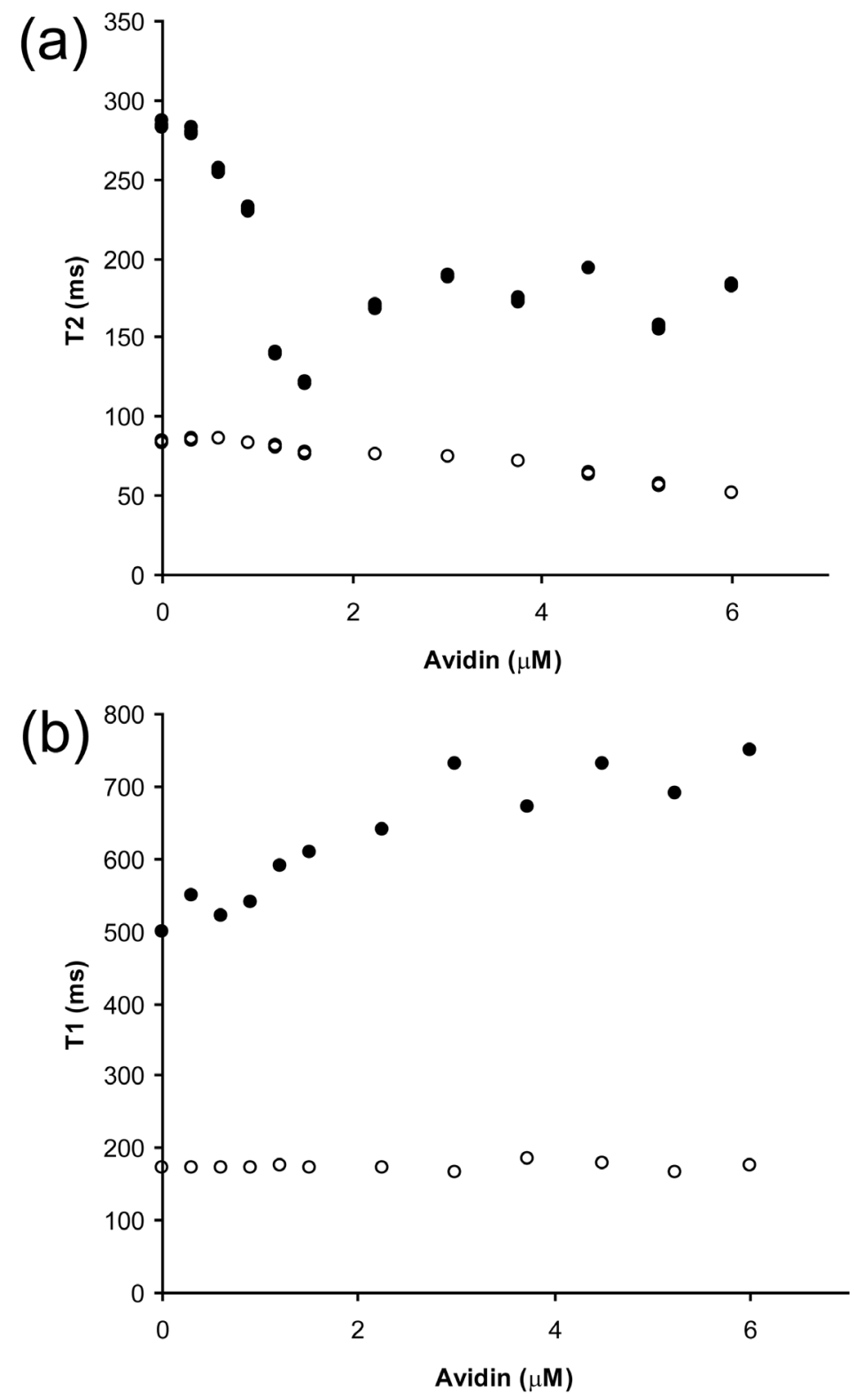

Figure S-3. Biotinylated crosslinked iron oxide nanoparticles (biotin-CLIO) were used as a nanoparticle system to demonstrate dose-response characteristics typical of MRSw diagnostics. (a) When avidin is added to a solution of $0.075 \mathrm{mM}$ Fe biotin-CLIO (closed circles), $\mathrm{T}_{2}$ decreases linearly with increasing avidin until $1.5 \mu \mathrm{M}$ avidin is reached, beyond which the $\mathrm{T}_{2}$ response is erratic, similar to that previously reported (Taktak et al. 2007). Addition of avidin to a solution of $0.25 \mathrm{mM}$ Fe biotin-CLIO (open circles) shows a smaller $T_{2}$ linear response over a broader range of analyte. (b) $T_{1}$ measurements of these same solutions shows that the dilute biotin-CLIO solutions are unstable after $\sim 1.5 \mathrm{mM}$ (closed circles) and concentration biotin-CLIO is stable over the complete range of avidin, suggesting that $0.25 \mathrm{mM} \mathrm{Fe}$ biotin-CLIO may be responsive over an even larger concentration range of avidin. Individual samples were measured sequentially inside the disposable plastic cartridge. $\mathrm{T}_{2}$ data was measured and reported in triplicate. 
(a)
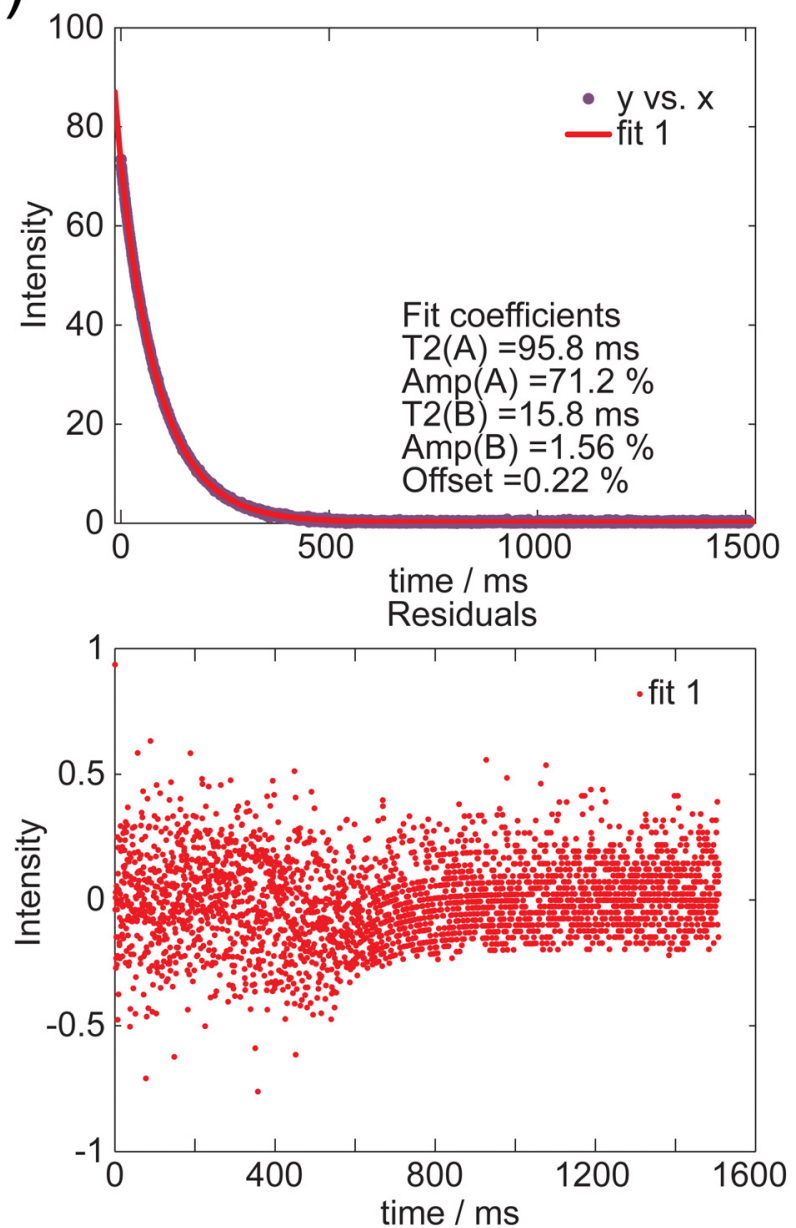

(b)
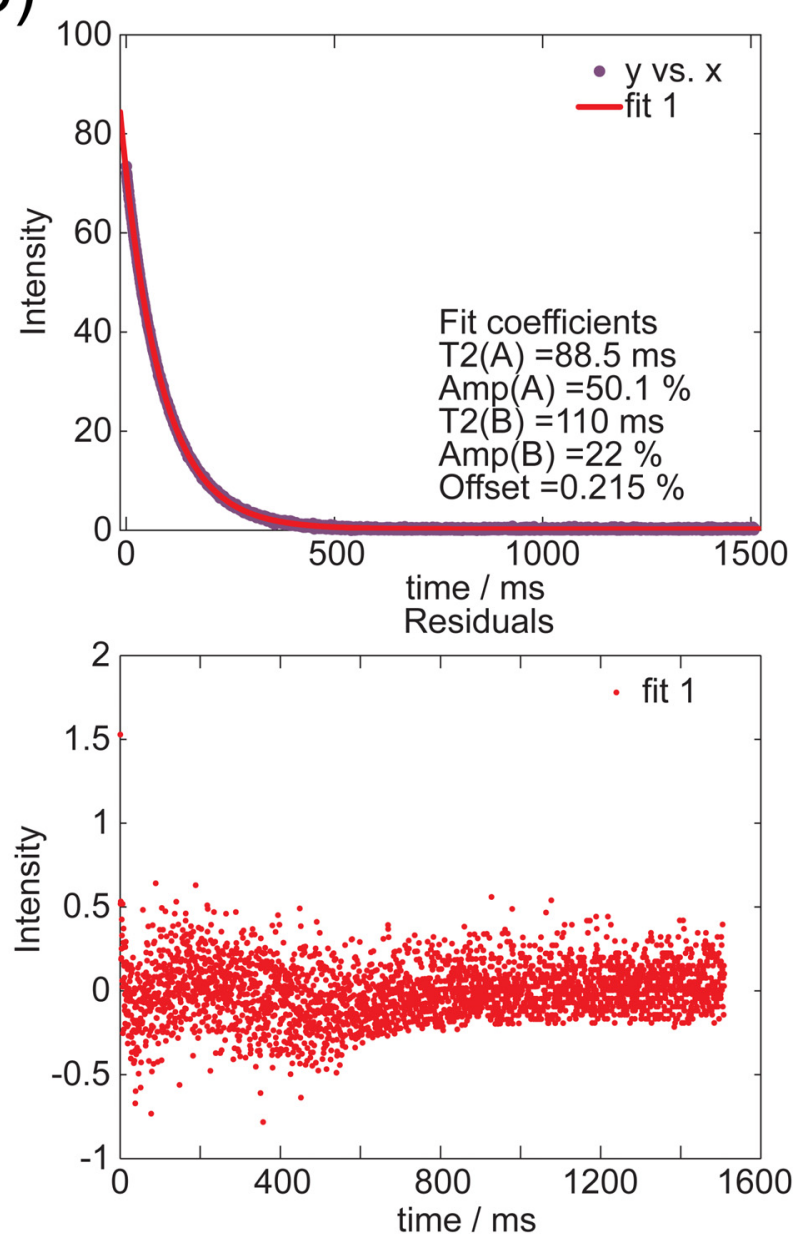

Figure S-4. Exponential relaxation curves for $\mathrm{CuSO}_{4}$ sample combination 9 (Figure 3) analyzed using CNNLS and (a) Equation (2) and (b) Equation (3). Fits and residuals demonstrate the similar quality of fit for both equations. The loss of resolution for both Equations (3) and (4) was not accompanied by a decrease in the quality of the fit as indicated by residual plots. 